Citation: S. Belotti (2021). Collezioni cartografiche e digital humanities per una nuova fruizione del patrimonio: il caso della Biblioteca Estense Universitaria di Modena. Bollettino della Società Geografica Italiana serie 14, 4(1): 95-107. doi: 10.36253/bsgi-1247

Copyright: (c) 2021 S. Belotti. This is an open access, peer-reviewed article published by Firenze University Press (http://www.fupress.com/bsgi) and distributed under the terms of the Creative Commons Attribution License, which permits unrestricted use, distribution, and reproduction in any medium, provided the original author and source are credited.

Data Availability Statement: All relevant data are within the paper and its Supporting Information files.

Competing Interests: The Author(s) declare(s) no conflict of interest.

\section{Collezioni cartografiche e digital humanities per una nuova fruizione del patrimonio: il caso della Biblioteca Estense Universitaria di Modena}

\author{
Map collections and digital humanities for a new access of \\ heritage: the case study of the Biblioteca Estense Universitaria di \\ Modena
}

\author{
SARA Belotti ${ }^{1}$ \\ Dipartimento di Studi Linguistici e Culturali, Università degli studi di Modena, Italia \\ E-mail: sara.belotti@unimore.it
}

\begin{abstract}
Digital humanities is an emerging discipline that has become increasingly popular in recent years, thanks to the implementation of numerous projects that aim at a dynamic dialogue between digital technologies and humanistic research. This is the scope of the project launched by the Biblioteca Estense Universitaria (BEU) di Modena in 2017, which, in collaboration with the University of Modena and Reggio Emilia, included the study, cataloguing and digitization of the cartographic collection, along with the music collection and the Muratorian collection. This project led to the creation of a digital library, inaugurated in June 2020, which not only allowed the enhancement of the cartographic collection, still little known, and to make it available, albeit only virtually, to scholars, but also led to the adoption of the IIIF protocol that allows to compare, edit, annotate and share the documents of the Este collection and collections that participate in the same circuit, providing new useful tools for research. In this context, the contribution, starting from the presentation of the Estense Digital Library project, presents the cartographic collection of the BEU and offers a reflection on the potential that the new digital media provide for the study of cartography and, more broadly, of heritage in the digital age.
\end{abstract}

Keywords: digital humanities, cartography, historical cartography, digital library, heritage.

Riassunto. Le digital humanities sono una disciplina emergente che negli ultimi anni si è andata sempre più diffondendo, grazie alla realizzazione di numerosi progetti che hanno come obiettivo il dialogo dinamico tra tecnologie digitali e ricerca umanistica. In tale ambito rientra il progetto avviato dalla Biblioteca Estense Universitaria di Modena (BEU) nel 2017 che, in collaborazione con l'Università di Modena e Reggio Emilia, ha previsto lo studio, la catalogazione e la digitalizzazione del fondo cartografico, insieme ad altri due fondi, quello musicale e quello muratoriano. Tale progetto ha

\footnotetext{
${ }^{1}$ Affiliazione attuale: Centro Studi sul Territorio, Università degli Studi di Bergamo, sara.belotti@ unibg.it.
} 
portato alla creazione di una digital library, inaugurata a giugno 2020, che non solo ha consentito di valorizzare la collezione cartografica, ancora poco conosciuta, e di renderla disponibile, seppure solo virtualmente, agli studiosi, ma ha portato anche all'adozione del protocollo IIIF che consente di confrontare, modificare, annotare e condividere i documenti della collezione estense e di collezioni che partecipano allo stesso circuito, fornendo nuovi strumenti utili per la ricerca. In tale contesto il contributo, partendo dalla presentazione del progetto Estense Digital Library, racconta la collezione cartografica della BEU e offre una riflessione sulle potenzialità che i nuovi mezzi digitali forniscono per lo studio della cartografia e, in modo più esteso, del patrimonio nell'era digitale.

Parole chiave: digital humanities, cartografia, cartografia storica, biblioteca digitale, patrimonio.

\section{Introduzione}

La rivoluzione digitale ha profondamente trasformato la società, con effetti rilevabili in ogni ambito professionale, sociale e culturale. In particolare, il mondo della comunicazione ha subito un importante rinnovamento, con il cambiamento delle regole che governano il settore, la trasformazione dei classici media e dell'editoria e la creazione di nuovi paradigmi sociali e culturali. Sono cambiate le tecnologie, i linguaggi espressivi e i contenuti, coinvolgendo anche numerose istituzioni, tra cui i musei e le gallerie d'arte, che oggi non espongono più solo opere d'arte "reali", ma anche installazioni digitali; o le biblioteche, che si sono dovute adattare alla sempre maggiore diffusione di $e$-book. In generale, il sistema culturale ha dovuto trovare nuovi modi di interagire con il proprio pubblico al fine di rispondere alla necessità di aprirsi all'innovazione, consentendo un maggiore accesso all'informazione. Questo processo di innovazione è stato ulteriormente incentivato con il dilagare della pandemia da Covid-19 che ha portato a lunghi periodi di chiusura dei servizi culturali, dei musei e delle biblioteche. Tali istituzioni, infatti, hanno cercato di reinventarsi, proponendo visite virtuali, nel caso dei musei, o letture e presentazioni di libri on-line, nel caso delle biblioteche, cui si è aggiunta la promozione di servizi e risorse web: cataloghi e biblioteche digitali (Lamberti, Tammaro 2020). Non solo, la rivoluzione digitale ha portato anche alla nascita di nuove discipline, tra cui le digital humanities che propongono una riflessione sul modo in cui trasmettere il sapere umanistico nell'era digitale. È proprio in tale ambito che si inserisce il progetto che ha sollecitato, proprio durante la pandemia (a fine giugno 2020), l'inaugurazione dell'Estense Digital Library (https://edl.beni- culturali.it/), ossia la biblioteca digitale della Biblioteca Estense Universitaria di Modena (BEU). La piattaforma è il frutto di un importante progetto di digitalizzazione del patrimonio iniziato nel 2017 dall'Estense, finanziato dalla Fondazione di Modena nell'ambito di AGO Modena Fabbriche Culturali e realizzato in partenariato con Università degli Studi di Modena e Reggio Emilia. La piattaforma apre la possibilità di consultare oltre 700 mila pagine di documenti, costituiti da libri antichi, mappe, documenti musicali, manoscritti e scritti di Ludovico Antonio Muratori. A ciò si aggiunge la possibilità di confrontare, modificare, annotare e condividere i documenti mediante il protocollo IIIF (International Image Interoperability Framework, https://iiif.io).

In tale contesto, l'articolo si concentra in particolare sul lavoro svolto sul patrimonio cartografico che ha portato alla catalogazione, allo studio e alla valorizzare del fondo consentendo non solo di mostrare al pubblico, seppur solo virtualmente, i più famosi documenti posseduti, tra cui troviamo la Carta del Cantino ${ }^{2}$, il Mappamondo catalano estense ${ }^{3}$ e il Planisfero Castiglioni ${ }^{4}$, ma ha anche permesso l'accesso a pubblico e studiosi a collezioni meno conosciute e ancora poco studiate, tra cui la collezione Forni. Il contributo, partendo dalla presentazione del progetto che ha portato alla pubblicazione dell'Estense Digital Library, si concentrerà sul fondo cartografico della BEU e sulle potenzialità che le digital humanities e i nuovi mezzi digitali forniscono per lo studio delle carte geografiche e, in modo più esteso, del patrimonio nell'era digitale.

\section{Il progetto Estense Digital Library}

Nel 2017 le Gallerie Estensi e la Biblioteca Estense Universitaria di Modena ${ }^{5}$ hanno avviato un importante

${ }^{2}$ Una delle prime mappe che rappresentano la costa del continente americano. La carta venne fatta realizzare da un cartografo portoghese da Alberto Cantino, inviato in Portogallo da Ercole I duca di Ferrara per raccogliere informazioni sulle nuove scoperte geografiche fatte da Spagna e Portogallo. L'opera venne poi spedita in Italia, contravvenendo alle norme portoghesi che vietavano la circolazione di documenti del genere (Milano 1991).

${ }^{3}$ Si tratta di una carta manoscritta su pergamena tonda di ampie dimensioni realizzata a metà del ' 400 presumibilmente dalla scuola catalana (Milano 2001).

${ }^{4}$ Grande carta nautica che rappresenta il mondo conosciuto nel 1525 . É attribuita a Diego Ribeiro, "piloto maior" della Casa de Contratación di Siviglia, l'ufficio della corona spagnola incaricato di rappresentare su mappe le nuove scoperte geografiche. La carta fu donata dall'imperatore Carlo V a Baldassare Castiglione, nunzio apostolico in Spagna e autore del celebre libro Il Cortegiano. Nell'estate del 2000 è stata acquistata dallo Stato italiano e destinata alla BEU (Milano 2002).

${ }^{5}$ La BEU fa oggi parte del complesso museale denominato Gallerie Estensi, nato a seguito della riforma del Ministero dei beni e delle attività culturali e del turismo del 2014. Il complesso include, oltre alla BEU, 
progetto di digitalizzazione e studio del proprio patrimonio. Tale progetto si inserisce in un contesto molto più ampio, che ha visto la Fondazione di Modena, finanziatore del progetto di digitalizzazione, impegnata negli ultimi anni nella creazione di AGO Modena Fabbriche Culturali e nel recupero dell'ex Ospedale di Sant'Agostino ${ }^{6}$, con lo scopo di creare un nuovo polo culturale per la città ${ }^{7}$.

In riferimento al progetto di digitalizzazione del patrimonio della $\mathrm{BEU}$, la prima fase ha previsto l'attivazione di tre assegni di ricerca a inizio 2018 in collaborazione con l'Università di Modena e Reggio Emilia dedicati a tre nuclei documentali di particolare importanza: il fondo cartografico; il fondo musicale e il fondo Muratoriano. I tre assegni di ricerca avevano l'obiettivo di identificare i documenti appartenenti ai tre nuclei e svolgere un lavoro di approfondimento, al fine di supportare da un punto di vista scientifico il lavoro di catalogazione e digitalizzazione che era previsto per la seconda fase di attività. Inoltre, il progetto ha portato al consolidamento della collaborazione che BEU e Gallerie Estensi hanno già da alcuni anni con il laboratorio AImageLab del Dipartimento di Ingegneria Informatica dell'Università di Modena e Reggio Emilia, che sta utilizzando le immagini digitalizzate per il training di alcuni algoritmi di machine learning.

la Galleria Estense e il Museo lapidario, a Modena, il Palazzo ducale di Sassuolo e la Pinacoteca nazionale di Ferrara.

${ }^{6}$ Nato come Grande Spedale degli Infermi tra il 1753 ed il 1758 per volere del Duca Francesco III d'Este il palazzo dell'ex Ospedale di Sant'Agostino si estende per oltre 40 mila metri quadrati. Ospita la Farmacia Storica ed il Teatro Anatomico (seconda metà del Settecento) recentemente restaurati. Nella stessa piazza, sul lato opposto, si trova il Palazzo dei Musei, in cui hanno sede la Biblioteca Estense Universitaria, le Gallerie Estensi, i Musei Civici di Modena, la Biblioteca Poletti, la Gipsoteca Giuseppe Graziosi, il Lapidario Romano, il Museo Lapidario Estense e l'Archivio storico Comunale. Sullo stesso lato della piazza si trova la Chiesa di Sant'Agostino, di origini trecentesche e trasformata nel 1669 nel Pantheon dei duchi d'Este. La chiesa, il teatro anatomico e la farmacia sono oggi visitabili grazie all'attività di AGO e alla collaborazione con l'Università di Modena e Reggio Emilia.

${ }^{7}$ L'ex Ospedale è diventato così un luogo di collaborazione per le istituzioni che operano negli spazi che si affacciano su Largo Porta Sant'Agostino, ma anche un luogo di dialogo con la città grazie alle numerose attività ed eventi. Inoltre, all'interno dellex Ospedale hanno sede il Centro Interdipartimentale di ricerca sulle Digital Humanities DHMoRe dell'Università di Modena e Reggio Emilia, il Future Education Modena (FEM), centro internazionale per la didattica innovativa, e la Fondazione Arti Visive. Per maggiori informazioni si veda: https://www.agomodena.it/it/.

${ }^{8}$ Nello specifico, tali attività rientrano nel progetto AI for Digital Humanities che vede il laboratorio impegnato in attività di media analysis, computer vision e pattern recognition, di comprensione di video e testi e del linguaggio naturale, di intelligenza artificiale e machine learning. In tale ambito diversi progetti hanno coinvolto le Gallerie Estensi e la $\mathrm{BEU}$, promuovendo la collaborazione tra ingegneri e ricercatori umanistici. Tra i progetti già realizzati Rerum Novarum, del 2010, che ha visto la digitalizzazione della Bibbia di Borso d'Este e la creazione di una
Nel 2019 è stato pubblicato un bando da un milione e trecento mila euro per il restauro, la digitalizzazione ad alta risoluzione e la catalogazione dei tre fondi già in fase di studio da parte degli assegnisti oltre che per il recupero del patrimonio oggetto delle campagne di digitalizzazione degli anni precedenti ${ }^{9}$. Il bando è stato vinto da una associazione temporanea di imprese denominata Extense, che ha come capofila Franco Cosimo Panini Editore, storica casa editrice modenese, incaricata del restauro, della fornitura del sistema di archiviazione digitale e delle digitalizzazioni dei documenti di grande formato, tra cui rientrano le mappe. Le altre aziende coinvolte sono Horizons Unlimites, che ha messo a disposizione la piattaforma $\mathrm{MLOL}^{10}$ per la realizzazione e la gestione della digital library; Hyperborea, che si occupa del coordinamento del progetto, del censimento dei nuclei documentali e della metadatazione tramite il software di catalogazione Arianna; infine, MIDA Informatica incaricata delle attività di digitalizzazione, escluso i grandi formati ${ }^{11}$.

Il lavoro è stato organizzato in due parti: una per il recupero delle digitalizzazioni pregresse e l'altra dedicata al materiale da digitalizzare ex novo. Il lavoro di recupero dei documenti già digitalizzati, in particolare, è stato sviluppato per poterne migliorare l'accessibilità. Le versioni digitali, infatti, erano consultabili solo tramite un pc fisso collocato nella sala di lettura della biblioteca e ogni documento era identificato unicamente con il numero di segnatura, senza ulteriori metadati. Ciò comportava, nella pratica, l'impossibilità di fare una ricerca nella cosiddetta Teca Digitale (circa 330.000 immagini) per parola chiave, titolo o autore, ma al contrario implicava la necessità di effettuare prima una ricerca

piattaforma multimediale multitouch per poter sfogliare virtualmente il famoso codice miniato quattrocentesco (https://aimagelab.ing.unimore. it/imagelab/pdf/20100924RerumNovarum.pdf).

${ }^{9} \mathrm{Il}$ primo progetto di digitalizzazione attivato in BEU risale all'inizio degli anni Novanta quando una serie di documenti e carte geografiche sono stati fotografati e le diapositive digitalizzate nell'ambito del progetto Leggio elettronico (1991-1995). Negli anni successivi sono state realizzate altre campagne di digitalizzazione, sebbene limitate a specifici fondi documentali (tra cui parte dell'archivio muratoriano nel 1999) o documenti particolarmente preziosi (tra cui la Carta degli stati estensi di Marco Antonio Pasi, a fine anni Novanta). Per un approfondimento riguardante i progetti di digitalizzazione portati avanti dalla $\mathrm{BEU}$ tra il 1991 e il 2010 si veda: Palazzi, 2010.

${ }^{10}$ MLOL (MediaLibraryOnLine) è la prima rete italiana di biblioteche pubbliche, accademiche e scolastiche per il prestito digitale che consente a tutti gli utenti iscritti a una delle biblioteche aderenti di consultare gratuitamente, attraverso il portale https://www.medialibrary.it/ e-book, musica, film, giornali, banche dati, corsi di formazione online (e-learning), archivi di immagini ecc. Ad oggi le biblioteche aderenti sono oltre 6.000 in 20 regioni italiane e 10 Paesi stranieri.

${ }^{11}$ Per maggiori informazioni riguardo i partner e il progetto si veda il sito: https://edl.beniculturali.it/. 
sui cataloghi cartacei o scansionati ${ }^{12}$ della biblioteca per poi procedere, una volta identificato il documento desiderato, alla consultazione della versione digitale (Zanni 2019). In generale, seppure alcuni dei documenti digitalizzati negli anni siano stati inseriti anche in piattaforme digitali, tra cui Internet Culturale, Europeana e Manus OnLine ${ }^{13}$, mancava una piattaforma unica che consentisse agli studiosi di tutto il mondo di accedere al patrimonio della BEU in modo immediato e semplice. Il lavoro sul materiale pregresso, quindi, ha previsto l'analisi e il censimento dei documenti presenti nella Teca Digitale, la normalizzazione delle segnature, il matching delle segnature con cataloghi e inventari esterni (Internet culturale, Manus, ecc.) per il recupero dei metadati e il rilevamento dei metadati minimi mancanti da parte di catalogatori specializzati (Zanni 2019).

Per quanto riguarda il lavoro sui nuovi documenti, invece, i tecnici hanno stilato gli elenchi dei materiali per poi confrontarsi con i ricercatori dell'Università di Modena e Reggio Emilia al fine di includere nel nuovo database le informazioni che stavano raccogliendo. Tale lavoro ha portato alla definizione dei tracciati per la metadatazione che hanno cercato di integrare le norme catalografiche e gli standard definiti dagli enti di setto$\mathrm{re}^{14}$ con le informazioni scaturite dal lavoro di ricerca

\footnotetext{
${ }^{12} \mathrm{Si}$ specifica che la BEU non possiede un catalogo unico del proprio patrimonio, ma sono disponibili diversi inventari e cataloghi parziali, realizzati in epoche e con modalità diverse, dedicati a specifici temi (per esempio i testi latini) o collezioni (per esempio la collezione Campori). Alcuni di questi cataloghi e inventari sono stati scannerizzati e sono consultabili in formato pdf, non ricercabile, sul sito della BEU.

${ }^{13}$ Internet Culturale è un portale web del Servizio bibliotecario nazionale, inaugurato nel 2005, che fornisce un punto comune di accesso per le risorse digitali e i cataloghi di biblioteche, archivi e istituzioni culturali italiane (https://www.internetculturale.it/). Europeana è una biblioteca digitale europea, nata nel 2008, che riunisce contributi già digitalizzati da diverse istituzioni dei ventotto Paesi membri dell'UE (https://www. europeana.eu/it). Manus è un database dei manoscritti in alfabeto latino prodotti dal Medioevo all'Età contemporanea conservati nelle biblioteche italiane. Il censimento è iniziato nel 1988 a cura dell'Istituto Centrale per il Catalogo Unico (ICCU) (https://manus.iccu.sbn.it/).

${ }^{14}$ La difficoltà di riconoscimento della mappa, che spazia dallo strumento geografico alla rappresentazione artistica/paesaggistica arrivando fino al progetto architettonico, pongono spesso questioni anche sulla sua catalogazione non consentendo una classificazione a priori. Nelle biblioteche questo materiale, spesso assimilato a stampe e disegni, più raramente raccolto in specifiche collezioni, viene catalogato in base all'International Standard Bibliographic Description for Cartographic Materials (International Federation fo Library Association and Insitutions, 1977, con aggiornamento nel 2007 e 2009) che fornisce indicazioni per la redazione delle schede descrittive che, però, spesso non sono esaustive, soprattutto nel caso delle carte manoscritte. Lo standard internazionale è stato recepito a livello nazionale tramite le Regole italiane di catalogazione per autore (1979), successivamente aggiornato con la pubblicazione delle nuove Regole italiane di catalogazione (REICAT) nel 2009 (https://www.iccu.sbn.it/it/attivita-servizi/gruppi-di-lavoro-e-commissioni/pagina_94.html). In molti archivi storici, viceversa, le mappe sono
}

che era in corso $^{15}$. A questo lavoro si è affiancato quello di valutazione dei materiali identificati da parte di una restauratrice al fine di valutarne lo stato di conservazione. I documenti valutati non idonei che presentavano lievi criticità sono stati stabilizzati, così da garantire la massima sicurezza nelle fasi di digitalizzazione, mentre quelli che richiedevano interventi di restauro di maggiore entità sono stati esclusi. La digitalizzazione ad altissima risoluzione ha adottato una politica di digital preservation in linea con i programmi Metamorfoze (Paesi Bassi - https://www.metamorfoze.nl/) e FADGI (USA) ${ }^{16}$ e ha rispettato lo standard ISO 19264-1. I master file dei singoli scatti sono archiviati nel formato DNG, mentre è stato utilizzato il formato TIFF per le ricostruzioni finali dei documenti. Tutte le immagini sono poi confluite nel DAM (Digital Asset Manager), ossia una infrastruttura di long term preservation, mentre i metadati descrittivi sono stati inseriti nel software Arianna ${ }^{17}$. La piattaforma Estense Digital Library, infine, è stata pubblicata tramite MLOL con la creazione di un front end, mediante il quale è possibile effettuare la ricerca di tutti i metadati descrittivi presenti nella scheda conservata in Arianna e visualizzare l'immagine corrispondente del DAM (Zanni 2019).

inserite all'interno di relazioni peritali o progetti territoriali e vengono catalogate seguendo gli standard per la descrizione delle serie e dei fondi documentali (General International Standard Archival Description e International Standard Archival Authority Record for Corporate Bodies, Persons and Famlies, prima edizione 1996), senza evidenziarne le peculiarità (Sgambati 2010).

${ }^{15}$ I progetti di ricerca Unimore, durati due anni dal 2018 al 2020, hanno subito lo scotto di non essere avviati in contemporanea con il progetto di digitalizzazione e creazione della digital library. I due progetti, infatti, andavano nella stessa direzione, ossia la catalogazione e valorizzazione del patrimonio, ma l'avvio della parte tecnica di digitalizzazione e progettazione quasi un anno dopo i progetti di ricerca ha portato a uno sfasamento del lavoro e un ricentramento delle attività che, nella seconda fase, si sono prettamente concentrate sulla catalogazione più che sulla ricerca anche da parte degli assegnisti. A tal proposito da parte dell'Università era stato creato, durante il primo anno di ricerca, un database grazie alla collaborazione con l'AImageLab del dipartimento di Ingegneria Informatica che avrebbe dovuto contenere la schedatura dei documenti da cui si sarebbe poi potuto accedere a schede di approfondimento (per esempio per la parte cartografiche a schede su cartografi, incisori, editori ecc.) con la creazione di una piattaforma che potesse essere qualcosa in più di un catalogo. Nella fase di lavoro in collaborazione con Extense, però, questo database è stato accantonato, al fine di soddisfare le cogenti esigenze di catalogazione, che sono diventate prioritarie con l'inizio della digitalizzazione e la concreta progettazione della digital library.

${ }^{16}$ Metamorfoze e Federal Agencies Digital Giudlines Initiatives (FADGI) sono due delle linee guida più note a livello internazionale che definiscono hardware, software, formati immagine e definizione dei flussi di lavoro per la digitalizzazione di documenti.

${ }^{17}$ Arianna è un software per la descrizione catalografica multi-ambito di oggetti culturali che consente di collegare la scheda descrittiva di ciascun oggetto alla sua riproduzione digitale. 
Il progetto ha previsto, oltre alla realizzazione di una biblioteca digitale ad accesso libero, l'attivazione di interfacce per l'annotazione e il crowdsourcing grazie al IIIF (International Image Interoperability Framework) ${ }^{18}$, un protocollo open source per la presentazione, ricerca e annotazione di immagini ad alta definizione che al momento della realizzazione del progetto ${ }^{19}$ non era ancora utilizzato da nessuna biblioteca italiana (Zanni 2019). IIIF consente lo scambio di digitalizzazioni fra istituzioni diverse collegandosi ai diversi database grazie a una URL che trasmette l'immagine e i metadati. In questo modo l'utente può, direttamente tramite Internet e in modo piuttosto semplice, confrontare i documenti digitalizzati appartenenti a più istituzioni, osservando anche il più piccolo dettaglio grazie alle funzioni di zoom e navigazione. È possibile creare, inoltre, un proprio account all'interno del quale costruire raccolte di documenti, editare le immagini con strumenti di photoediting o ancora inserire annotazioni su di un'immagine o sue porzioni con testo libero e tag (Di Giammarco 2019). In particolare, le annotazioni diventano esse stesse un oggetto digitale, con URL e coordinate dell'immagine ritagliata che, in fase successiva, possono essere condivise nella piattaforma stessa oppure tramite social media o siti web.

\section{Il fondo cartografico della Biblioteca Estense Universitaria di Modena}

La collezione cartografica posseduta dalla Biblioteca Estense Universitaria di Modena si compone di documenti prodotti tra il XV e l'inizio del XX secolo e si caratterizza per la presenza di alcuni preziosi manoscritti, di antiche incisioni in rame e stampe più moderne. L'estensione temporale coperta ci consente non solo di apprezzare il mutamento nella tecnica di rappresentazione del territorio, ma anche di ricostruire le diverse visioni del mondo che hanno caratterizzato i secoli passati ${ }^{20}$.

\footnotetext{
${ }^{18}$ IIIF nasce nel 2011 da un consorzio di importanti biblioteche, tra cui Stanford University, British Library, Bodleian Libraries, Bibliothèque national de France, Nasjonalbiblioteket, Los Alamos National Library, Cornell University, ed è diventato in pochi anni uno standard per le istituzioni culturali di tutto il mondo.

${ }^{19}$ La prima biblioteca a pubblicare in Italia una digital library con la possibilità di utilizzo dello standard IIIF è l'Ambrosiana di Milano, che ha inaugurato la sua biblioteca digitale a novembre 2019 (https://www. ambrosiana.it/partecipa/mostre-e-iniziative/la-nuova-biblioteca-digitaledella-veneranda-biblioteca-ambrosiana/).

${ }^{20} \mathrm{~A}$ tal proposito dal 22 settembre 2018 al 06 gennaio 2019 presso la BEU è stata organizzata una mostra dal titolo Meravigliose Avventure. Cartografia tra nuovi e vecchi mondi, curata dallautrice, che attraverso le mappe e gli atlanti posseduti dalla Biblioteca ripercorreva la storia della cartografia fino al XIX secolo.
}

Attraverso la cartografia possiamo analizzare le strutture paesistiche e territoriali, oltre che gli eventi connessi con la sfera politica, economica e sociale che hanno portato a una specifica configurazione territoriale, consentendoci di ricostruirne anche la percezione che ne avevano i contemporanei, così come di definire la scala di valori cui una società si attiene per interpretare il mondo in cui vive (Casti 1998).

La collezione oggi custodita in Estense ha avuto una lenta costituzione, avendo origine nell'interesse dei duchi d'Este verso la geografia e la conoscenza del mondo. Tra gli scaffali della biblioteca ducale, infatti, già dal XIV secolo era possibile trovare opere di famosi viaggiatori, tra cui Marco Polo e Mandeville, mentre gli illustri autori che frequentavano la corte, tra cui Ariosto e Boiardo ${ }^{21}$, davano vita ad alcuni dei più importanti romanzi cavallereschi in cui venivano narrati avventurosi viaggi veicolando un'immagine "fantastica" dell'altrove (Donattini 2000). Successivamente l'esplorazione dell'altrove diventa reale, grazie alla pratica del pellegrinaggio, a partire da quello organizzato da Niccolò III d'Este in Terra Santa nel 1413 con obiettivi al tempo stesso religiosi e politici (Bagnoli, Battini 2018). Nello stesso periodo, grazie all'impulso dato dal principe portoghese Enrico il Navigatore, si apre la via alle esplorazioni negli Oceani, dando vita a un importante processo di ridefinizione dello spazio che suscita interesse in tutti i potenti europei, inclusa la corte ferrarese.

É così che entrano a fare parte della biblioteca di corte numerose opere a tema geografico, tra cui spicca una famosa copia della Geografia di Tolomeo dedicata a Borso d'Este nel 1466 e ancora oggi presente in Estense (Bertoni 1903), a cui si affiancano man mano anche opere cartografiche, oggi di inestimabile valore. Un catalogo del 1471 cita tra gli arredi della biblioteca un mappamondo conservato in un guaina di cuoio, riferendosi presumibilmente al Mappamondo catalano estense, mentre è testimoniata da alcune lettere ancora oggi conservate presso l'Archivio di Stato di Modena l'impresa di Alberto Cantino che, nel 1502, porta a Ercole I una mappa in cui sono riportate le più recenti scoperte, nonostante il divieto assoluto, imposto dal sovrano portoghese, di portare fuori dai confini nazionali carte geografiche, pena la condanna a morte (Milano 1995;1991).

\footnotetext{
${ }^{21}$ Matteo Maria Boiardo, nato a Scandiano (RE), si trasferì a Ferrara nel 1461 andando a vivere alla corte di Borso d'Este e poi di Ercole I, fu governatore di Modena e poi di Reggio dove rimase fino alla morte. Ludovico Ariosto, nato a Reggio Emilia, si trasferì a Ferrara all'età di dieci anni, dove il padre Niccolò era funzionario al servizio dei duchi d'Este e comandante della guarnigione militare di Reggio Emilia. Ippolito d'Este, figlio di Ercole I d'Este, Duca di Modena e Ferrara fu un suo famoso mecenate a cui è dedicato l'Orlando furioso (1516).
} 
In termini culturali, l'interesse degli Estensi verso le nuove conoscenze geografiche e la cartografia rappresenta un mezzo importante per affrontare la sfida della marginalità spaziale e politica di Ferrara nel momento in cui, a seguito dell'apertura delle esplorazioni degli Oceani, il Mediterraneo perde la sua centralità economica (Salmons, Moretti 1984). Le mappe assumono così un ruolo strategico, poiché riassumono le notizie portate in Europa da esploratori, navigatori e mercati, oltre a fornire informazioni pratiche, come quelle riguardanti i poteri politici dei nuovi territori in esplorazione o le risorse economiche che poteva essere importate in Europa (spezie, pietre preziose, stoffe ecc.).

Con la morte di Alfonso II, nel 1598, e la successiva devoluzione del Ducato di Ferrara alla Santa Sede ${ }^{22}$, Modena divenne la nuova capitale dei domini estensi, dove vennero trasferiti tutti i beni dei duchi, inclusa la loro biblioteca che subì a partire da questo momento circa un secolo di totale oblio e di continue peregrinazioni da una sede all'altra. Tale situazione comportò enormi perdite e una pessima conservazione, fino a quando, nella seconda metà del Seicento Francesco II nominò dei nuovi bibliotecari.

Tanti furono i nomi illustri che da questo momento si susseguirono, tra cui si ricordano Ludovico Antonio Muratori e Girolamo Tiraboschi, ma due sono i nomi che spiccano non solo per il loro lavoro presso la biblioteca, ma anche per la loro opera cartografica, di cui ancora oggi troviamo testimonianze nella collezione dell'Estense. Il primo è Giovan Battista Boccabadati, dal 1671, insigne scienziato, giurista e letterato, nonché ingegnere di corte che realizzò, tra le altre, una dettagliata mappa della città di Modena nel 1684. La mappa è oggi conservata presso l'Archivio Storico del comune di Modena, mentre una copia realizzata nel secolo successivo da Domenico Vandelli si trova presso la Biblioteca Estense Universitaria, a testimonianza dell'importanza che questa mappa ebbe nella rappresentazione della città fino alla fine del Settecento, quando vari interventi promossi da Francesco III e Ercole III ne modificheranno la struttura (Comune di Modena 2009).

Il secondo bibliotecario fu Giacomo Cantelli, nominato anche geografo di corte nel 1685. Di Cantelli in

\footnotetext{
${ }^{22}$ Il 27 ottobre 1597 moriva il duca Alfonso II d'Este senza lasciare discendenti diretti. Il titolo di duca passò a Cesare, suo cugino, del ramo estense dei marchesi di Montecchio. La legittimità della successione, però, non venne riconosciuta dalla Chiesa, poiché la concessione papale di Ferrara agli Estensi limitava la continuità della signoria della casata ai soli discendenti diretti legittimi. Fu così che, a seguito di un accordo tra il Papa e il nuovo duca, le cosiddette Convenzioni Faentine, Ferrara e i territori della Romagna tornarono sotto il controllo dello Stato Pontificio, retto da Clemente VIII, mentre Modena divenne la nuova capitale del ducato.
}

BEU si conservano ancora numerose mappe, oltre ad alcune copie del suo Mercurio geografico, stampate a Roma nell'officina De Rossi e realizzate appositamente per i duchi estensi, come testimoniano le lettere conservate presso l'Archivio di Stato di Modena ${ }^{23}$.

Fu però solo successivamente, nel 1696 quando venne nominato bibliotecario padre Benedetto Bacchini che tutto il complesso librario e documentario venne sistemato nel Palazzo Ducale, costruito nel 1634 sul sito dell'antico castello. Qui fu conservata anche la collezione di carte geografiche fino al 1859, quando Francesco V d'Este abbandonò Modena a causa dell'arrivo dell'esercito del re Vittorio Emanuele II di Sardegna e della successiva annessione del ducato al Regno d'Italia. I tumulti scatenati dalla situazione travolsero anche il Palazzo Ducale e la sua biblioteca, da cui furono sottratte numerose carte geografiche, tra cui il Mappamondo catalano estense e la Carta del Cantino. Tali documenti vennero ritrovati solo undici anni dopo da Giuseppe Boni, probabilmente un collezionista modenese di cimeli geografici, il quale li donò all'allora Regia Biblioteca Estense dove sono ancora oggi conservati ${ }^{24}$.

L'annessione del Ducato al Regno di Sardegna nel 1860 segna una nuova fase per la Biblioteca che viene acquisita dal nuovo governo nazionale e trasferita nel 1882 nell'attuale sede di Palazzo dei musei, a cui si aggiunse, nel 1891, anche il patrimonio della Biblioteca Universitaria $^{25}$. A partire da questo momento la BEU diventa oggetto di numerose donazioni da famiglie modenesi a testimonianza del forte legame che questa istituzione aveva con la città. Grazie a tali donazioni, anche la raccolta di carte geografiche si amplia.

Le mappe che fanno parte del patrimonio dell'Estense sono oggi incluse in diversi cataloghi e inventari, a causa della mancanza di un catalogo unico. Seppure non sia semplice ricostruire la collezione cartografica ducale originaria, molte delle mappe appartenute ai duchi sono conservate nel cosiddetto "fondo estense", custodito nella sala manoscritti e incluso nell'Inventario dei portolani, delle carte nautiche e geografiche della $R$. Biblioteca Estense, trascritto nel 1892 da Clemente Laz-

\footnotetext{
${ }^{23}$ Per un approfondimento si veda: Bonazzi et al. 1995. In particolare nell'Appendice sono state trascritte, a cura della Biblioteca comunale F. Selmi di Vignola, paese natale del Cantelli, tutte le lettere autografe presenti all'Archivio di Stato di Modena e nella Biblioteca Estense Universitaria.

${ }^{24}$ In BEU è conservata la lettera del Boni con cui, in data 25 aprile 1870, sono stati donati i documenti.

${ }^{25} \mathrm{La}$ Biblioteca dell'Università venne fondata nel 1772 per volere di Francesco III d'Este. Il patrimonio delle due istituzioni venne riunito nel 1891, mentre i due enti vennero formalmente fusi solo nel 1995, con la creazione dell'attuale Biblioteca Estense Universitaria (http://bibliotecaestense.beniculturali.it/info/beu/storia.html).
} 
zarini, in parte ampliato a seguito di alcune acquisizioni successive. Oltre alle mappe già menzionate, tra i documenti sono di particolare interesse le quattro mappe manoscritte che compongono l'atlante di Jean-François Roussin (Milano 1991, 220-224) e le quattro carte nautiche manoscritte del XV secolo già descritte dall'Almagià nel 1926 (Almagià 1926), a cui si affiancano numerose altre mappe a scala locale, nazionale e globale, sia manoscritte sia a stampa.

Le carte che sono entrate a far parte della BEU dopo l'Unità d'Italia, invece, sono incluse negli inventari delle rispettive collezioni. Tra queste, ricordiamo la raccolta del marchese Giuseppe Campori, letterato e politico modenese, depositata in Biblioteca nel 1893, all'interno della quale troviamo pochi, ma preziosi esempi di cartografia nautica del secolo XVII e atlanti nautici manoscritti (Fabretti 1978; Milano 2001, 200-207; Milano 1991, 224-225). Nel 1920 arriva in Biblioteca la collezione del professor Chiaffredo Hugues, noto esponente modenese e cultore degli studi storico-artistici, in cui troviamo oltre cento mappe topografiche e piante di città, per la maggior parte risalenti al XIX secolo, prodotte dai principali istituti topografici dell'epoca, quali il Regio Istituto geografico militare austriaco e l'Istituto Geografico Militare, precedentemente denominato Ufficio Tecnico del Corpo di Stato maggiore del Regio Esercito e Istituto Topografico Militare. Tra il 1945 e il 1946 un'altra collezione arricchisce l'Estense, ossia quella della biblioteca di Antonio Bertacchini-Mattioli, un fondo di piccole dimensioni che include diciotto mappe prodotte nella seconda metà del XIX secolo, alcune stampate a Gotha (Germania) dalla Justus Perthes famosa casa editrice di carte geografiche dell'epoca.

Nel 2007 la Biblioteca riceve il deposito della biblioteca della famiglia Forni, i cui esponenti furono stretti collaboratori degli Estensi. Il nucleo originario della raccolta risale al chierico modenese Matteo Pagliaroli (1716-1777) che nella prima metà del XVIII secolo formò una ricca biblioteca, poi passata ai conti Forni per via successoria e nel tempo ampliata con nuove accessioni (Tinti 2014). Questa raccolta è particolarmente ricca di carte geografiche, suddivise in due nuclei. Il primo è composto da quattro album rilegati nel 1834 da Francesco Vignoli in cui sono state inserite o incollate circa 250 mappe prodotte da diversi cartografi tra XVIII e XIX secolo "per meglio conservarle, ed usarne», come riportato sul frontespizio del primo volume. Alcuni dei fogli che compongono la collezione sono stati estratti o ritagliati da altri volumi, tra cui l'Atlante novissimo pubblicato da Antonio Zatta a Venezia e l'Egeo redivivo che riporta un'estesa e dettagliata descrizione delle isole dell'Egeo, compilata da Francesco Piacenza su incarico del Duca di Modena Francesco II, a seguito delle vittorie del Morosini proprio in quest'area. Il secondo nucleo è costituito principalmente da carte topografiche, piante e vedute di città realizzate nel XIX secolo. Questa parte della collezione è attualmente in fase di catalogazione ed è stata solo parzialmente digitalizzata.

Nel tempo, alle donazioni si sono affiancati anche importanti acquisti, come la raccolta Pio Falcò di Savoia, entrata in Estense nel 2001, o la Carta Castiglioni acquistata nel 2000. Nello specifico, la prima è la biblioteca di casa Falcò Pio di Savoia che si trovava a Villa FalcòMombello di Imbersago (Lecco), venduta nel 1999 da Prospero Colonna, nipote ed erede del principe Alfonso Falcò Pio di Savoia, ultimo discendente della casata. Acquistata dal Ministero per i Beni e le Attività culturali venne destinata all'Estense, grazie al lavoro di Ernesto Milano, allora direttore della Biblioteca, che mise in luce i legami tra gli Este e i Pio di Carpi, poi Pio di Savoia. Nella collezione sono presenti poco più di dieci mappe realizzate tra la fine del XVII secolo e la fine del XIX secolo, che mostrano sia regioni europee, tra cui i regni di Navarra, Castiglia e Andalusia di Giacomo Cantelli, sia parti del territorio italiano settentrionale, tra cui alcune mappe stampate nel 1885 da Vallardi Editore.

La Carta Castiglioni fu realizzata nel 1525 e, secondo la tradizione, fu donata dall'Imperatore Carlo V a Baldassarre Castiglioni, valente uomo d'armi e letterato, nunzio apostolico in Spagna, in segno di benevolenza. Fu custodita dai marchesi Castiglioni per cinque secoli e raramente mostrata al pubblico. Acquistata dal Ministero per i Beni e le Attività culturali nell'anno 2000, il 28 luglio fece ufficialmente il suo ingresso alla BEU. Essa è uno dei documenti più importanti posseduti oggi dalla Biblioteca, è un esempio dei cosiddetti "padrones reales", carte che venivano costantemente aggiornate in base alle notizie che provenivano dai viaggi di esplorazione, gelosamente custodite dalla corona spagnola per evitare episodi di spionaggio commerciale.

Infine, alle collezioni fino a qui presentate, si aggiungono due serie di carte: le Piante di Modena $e$ dello Stato in cui sono state raggruppate, probabilmente a fini conservativi da parte della Biblioteca, circa una trentina di mappe della città e della provincia e la Serie Atlantica, verosimilmente proveniente dalla Biblioteca Universitaria che contiene circa sessanta mappe di diverse parti del mondo, realizzate tra il XVIII e il XIX secolo da vari autori, tra cui Guillaume De L'Isle.

Nel complesso la collezione include oggi circa un migliaio di mappe in fogli sciolti, per la maggior parte già digitalizzati, a cui si aggiungono numerosi atlanti che non sono stati presi in considerazione nel presente progetto, a dimostrazione dell'interesse che la cartogra- 
fia e, più in generale, la geografia hanno sempre suscitato nei secoli, sia per i duchi Estensi sia per altre famiglie nobili.

\section{Nuove opportunità di ricerca e valorizzazione nell'era delle digital humanities: dalla collezione cartografica alla digital library}

In campo umanistico ci si sta molto interrogando sugli impatti e le potenzialità che le digital humanities possono avere sulla ricerca e sullo studio delle discipline; si stanno cercando anche di sviluppare strumenti utili che, proprio grazie alla potenza e alla flessibilità del digitale, consentano oggi di fare cose fino a poco tempo fa impensabili. Le potenzialità di uno strumento come l'OCR (Optical Character Recognition), ossia il riconoscimento ottico dei caratteri, per esempio, ci ha consentito di rendere ricercabili testi stampati semplicemente attraverso l'uso di uno scanner ${ }^{26}$. Ciò ci permette oggi di fare ricerche per parole chiave, identificare termini ricorrenti, analizzare i testi e comparare più edizioni, in tempi molto più brevi di quelli necessari pre-era digitale. Le applicazioni delle digital humanities, però, non si limitano solo a questo, ma includono la creazione di mappe interattive, le ricerche di data e text mining, la creazione di indici lessicali ed edizioni critiche, ecc., favorendo un dialogo dinamico tra tecnologie emergenti e ricerca umanistica, così come tra discipline diverse, poiché "il digitale aggrega ed esalta l'interdisciplinarietà e la trasversalità delle ricerche, offrendo strumenti e metodologie comuni" (Cassella 2017).

L'innovazione maggiore che ha impattato sulla ricer$\mathrm{ca}$, però, è proprio la digitalizzazione dei documenti in sé, ossia la creazione di repertori documentali che possono essere consultati senza la necessità di recarsi nel luogo in cui vengono conservati e possono essere condivisi in pochi click. In passato, infatti, per poter studiare da vicino un manoscritto era necessario recarsi presso la biblioteca in cui era custodito e affrontare una serie di questioni burocratiche per poterlo sfogliare. Oggi, al contrario, la creazione di biblioteche digitali ci consente di accedere allo stesso codice comodamente da casa. Inoltre, l'uso di soluzioni ad altissima risoluzione ci permette di zoomare i documenti in modo agevole, anche con ingrandimenti che vanno al di là delle capacità dell'occhio umano (Mattei 2015). Vero è che la bellezza

\footnotetext{
${ }^{26}$ L'OCR fa parte dei campi di ricerca dell'intelligenza artificiale, della visione artificiale e del pattern recognition. I programmi OCR consentono di convertire in modo automatico un'immagine che contiene testo in un file digitale editabile.
}

di un documento manoscritto non può essere certo trasmessa attraverso lo schermo di un computer e l'emozione di avere tra le mani un documento realizzato centinaia di anni fa non è comparabile a quella che si prova mentre si naviga digitalmente tra le sue pagine, ma se consideriamo la fragilità di questo patrimonio e la difficoltà, in alcuni casi, ad accedere alla sua versione fisica, possiamo presto concludere che lo strumento digitale ha oggi una grande importanza per il lavoro del ricercatore. Nello specifico, in questo momento storico caratterizzato dalla pandemia da Covid-19, con la chiusura di numerose istituzioni, musei, archivi e biblioteche, la possibilità di accedere in modo virtuale ai cataloghi, agli inventari, ai documenti e alle pubblicazioni è stato essenziale per supportare la ricerca e consentirne la sua continuazione, nonostante le difficoltà.

In tale contesto, lo sviluppo di strumenti come quello dell'Estense Digital Library (EDL) risulta essere interessante. Con uno specifico riferimento alla collezione cartografica, come abbiamo già visto, il progetto ha portato alla digitalizzazione di più di seicento documenti appartenenti a numerose collezioni della BEU. Molte di queste sono ancora oggi poco studiate e poco conosciute, quindi il loro inserimento in EDL ne ha consentito la valorizzazione e la divulgazione, mettendole a disposizione degli studiosi. Non solo, con EDL è stato costruito un catalogo unico del patrimonio cartografico, finora inesistente, fornendo ai ricercatori uno strumento agile di ricerca.

Inoltre, la piattaforma EDL è suddivisa in due sezioni: Catalogo Estense e Catalogo Open. Il primo consente di fare la ricerca per parole chiave all'interno del database della BEU (circa 8.500 documenti tra mappe, manoscritti, libri a stampa e documenti musicali), mentre il Catalogo Open include più di un milione di documenti presenti in altre biblioteche digitali che possono essere consultati in modalità libera, ampliando l'offerta della BEU. A ciò si aggiunge il protocollo IIIF che, come già anticipato permette di creare annotazioni sui documenti e raccolte personalizzate. La funzione di base, per tutti coloro che accedono al sito e senza necessità di registrazione, è il visualizzatore, che permette di zoomare e navigare i documenti al fine di apprezzarne anche i più piccoli dettagli, grazie alle immagini ad altissima risoluzione. Nello specifico, questa funzione è molto utile nel caso delle mappe, in quanto consente di ingrandire i dettagli consentendone una più agevole lettura. Per esempio, nella Mappa del Cantino, che misura 2200x1050 mm, sono presenti numerosissimi designatori lungo le coste europee e africane a cui si aggiungono diverse legende che descrivono i territori rappresentati. Tali scritte ad occhio nudo sono leggibili, ma non sem- 


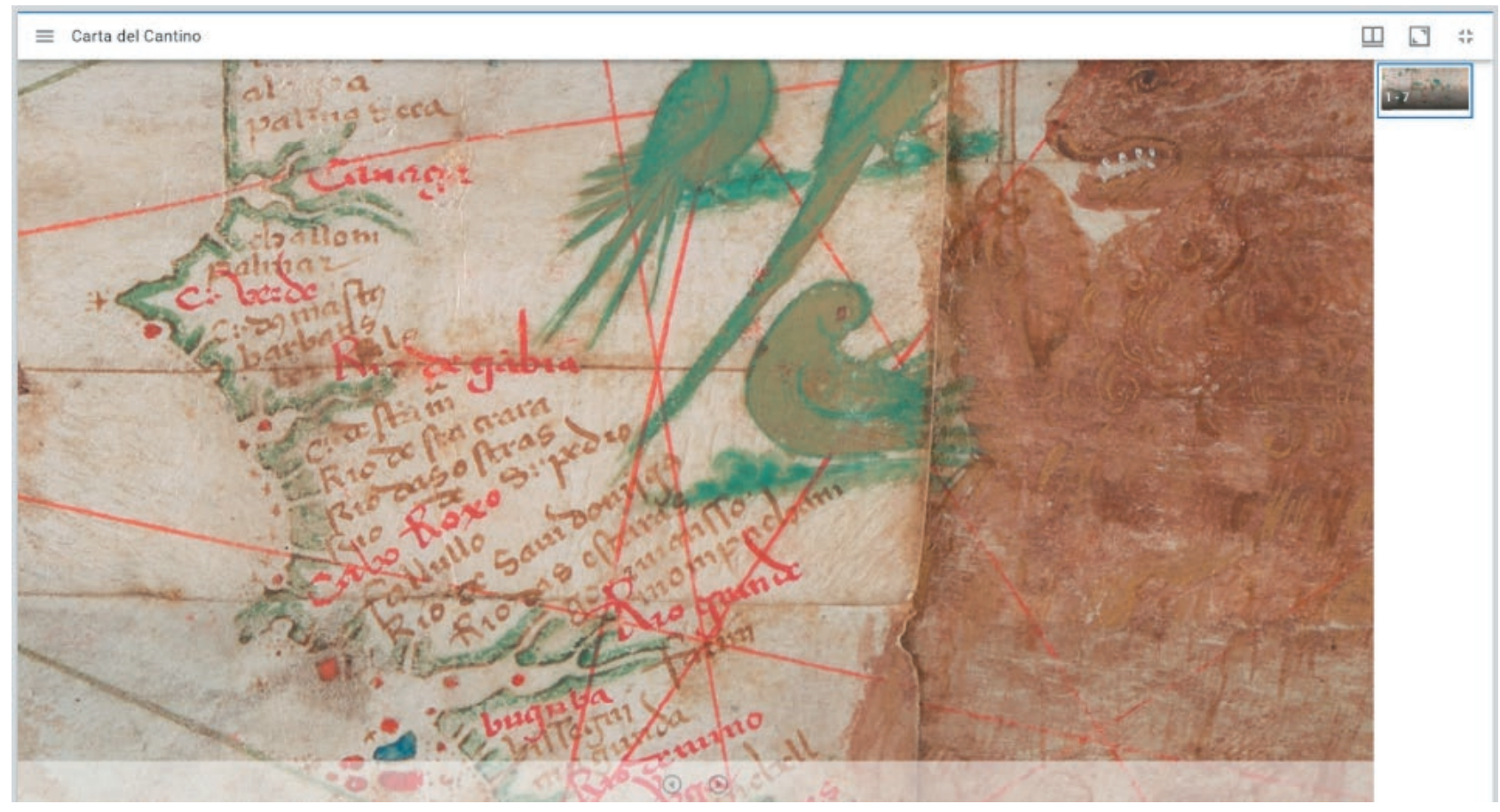

Figura 1. Esempio di zoom sulla Mappa del Cantino nel visualizzatore IIIF della costa africana. Fonte: Estense Digital Library.

pre facilmente, a ciò si aggiunge il fatto che la mappa è conservata in un'apposita teca, in posizione orizzontale, e, a causa delle sue dimensioni, risulta poco agevole da consultare. Il visualizzatore di IIIF, quindi, consente di avere una migliore visione e di apprezzare a pieno la rappresentazione (Fig. 1).

Per gli utenti registrati a questa funzione di base si aggiunge la possibilità di utilizzare altri strumenti per annotare, modificare e ritagliare le immagini digitalizzate. Le annotazioni sono una sorta di post-it che possono essere inseriti in diversi punti dell'immagine. Ad una prima azione, cliccando sul tasto "annota" il sistema richiede la creazione di un quaderno digitale in cui verranno salvate tutte le note, mentre la funzione "annota pagine" consente di annotare specifici dettagli del documento selezionando un'area, una linea o un punto. Per ognuno di questi oggetti è poi possibile inserire un titolo, un breve testo descrittivo e dei tag, ossia parole chiave che descrivono la nota, utilizzate per creare elenchi di annotazioni sullo stesso argomento in uno o più documenti (Fig. 2). Se l'utente desidera può scegliere di rendere le proprie annotazioni pubbliche, ciò non significa che saranno visibili a tutti coloro che consulteranno il documento, ma saranno visibili solo da coloro che accederanno alle liste di documenti rese pubbliche dall'utente. Oltre all'annotazione, dal visualizzatore IIIF è possibile attivare i tasti di photoediting per modifica- re la saturazione, la luminosità e il contrasto, e di ritaglio, mediante il quale creare dei ritagli di alcune parti dell'immagine che poi possono essere condividere tramite e-mail o social grazie al link che viene fornito dal sistema (Fig. 3).

Altra opzione è quella di creazione di Liste personalizzate di documenti selezionabili sia nel Catalogo Estense sia nel Catalogo Open che possono essere condivise tramite link con collaboratori o studenti. Inoltre, è possibile, tramite il visualizzatore, confrontare tra loro i documenti inclusi nelle liste. Tale strumento ha grandi potenzialità per lo studio e la ricerca, poiché da un lato è possibile creare una collezione di mappe personalizzata (o di mappe ed altri tipi di documenti), ricostruendo per esempio raccolte o pubblicazioni smembrate, dall'altro consente di comparare agevolmente diversi documenti. Nel nostro caso specifico è possibile confrontare mappe che rappresentano gli stessi territori, ma magari prodotte in periodi diversi, oppure più edizioni della stessa carta, oppure ancora è consentito affiancare rappresentazioni cartografiche e documenti testuali di descrizione. Confrontare collezioni appartenenti ad istituzioni diverse offre la possibilità di fare un lavoro di analisi che difficilmente si può fare con le versioni originali, se non con molte visite alle varie biblioteche (Fig.4). IIIF, al contrario, permette di affiancare in una stessa finestra più mappe, di sfruttare gli strumenti di zoom per vedere i 


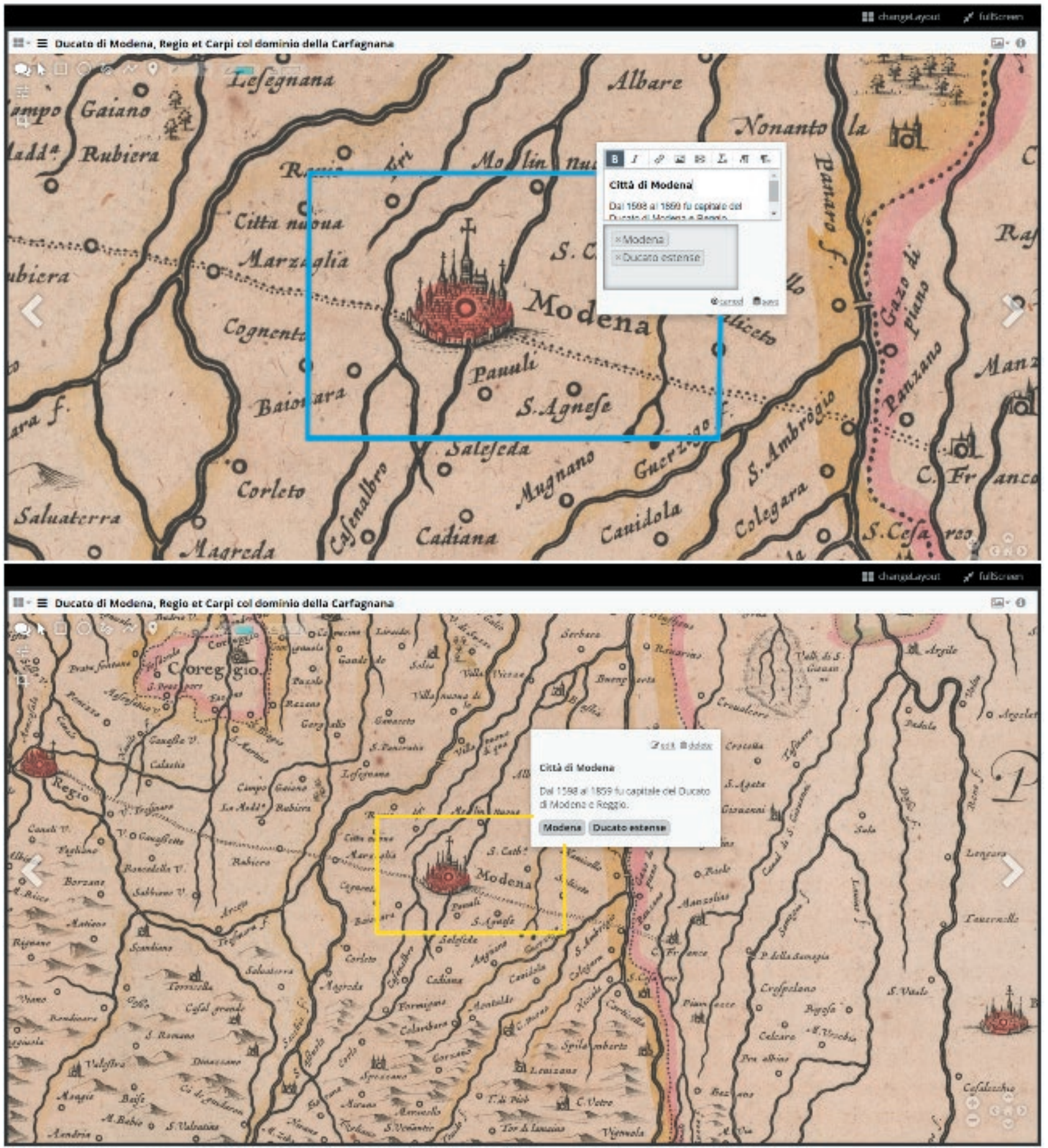

Figura 2. Esempio di didascalia inserita sulla mappa del Ducato di Modena e Reggio di Willem Janszoon Blaeu. In alto il form per la compilazione dell'annotazione; in basso la visualizzazione finale dell'annotazione. Fonte: Estense Digital Library.

dettagli e le funzioni di annotazione per prendere nota delle osservazioni.

Infine, un altro strumento fornito direttamente da IIIF è quello delle Storie, che consente di creare una presentazione direttamente navigando all'interno del documento digitale, selezionando specifiche porzioni, 


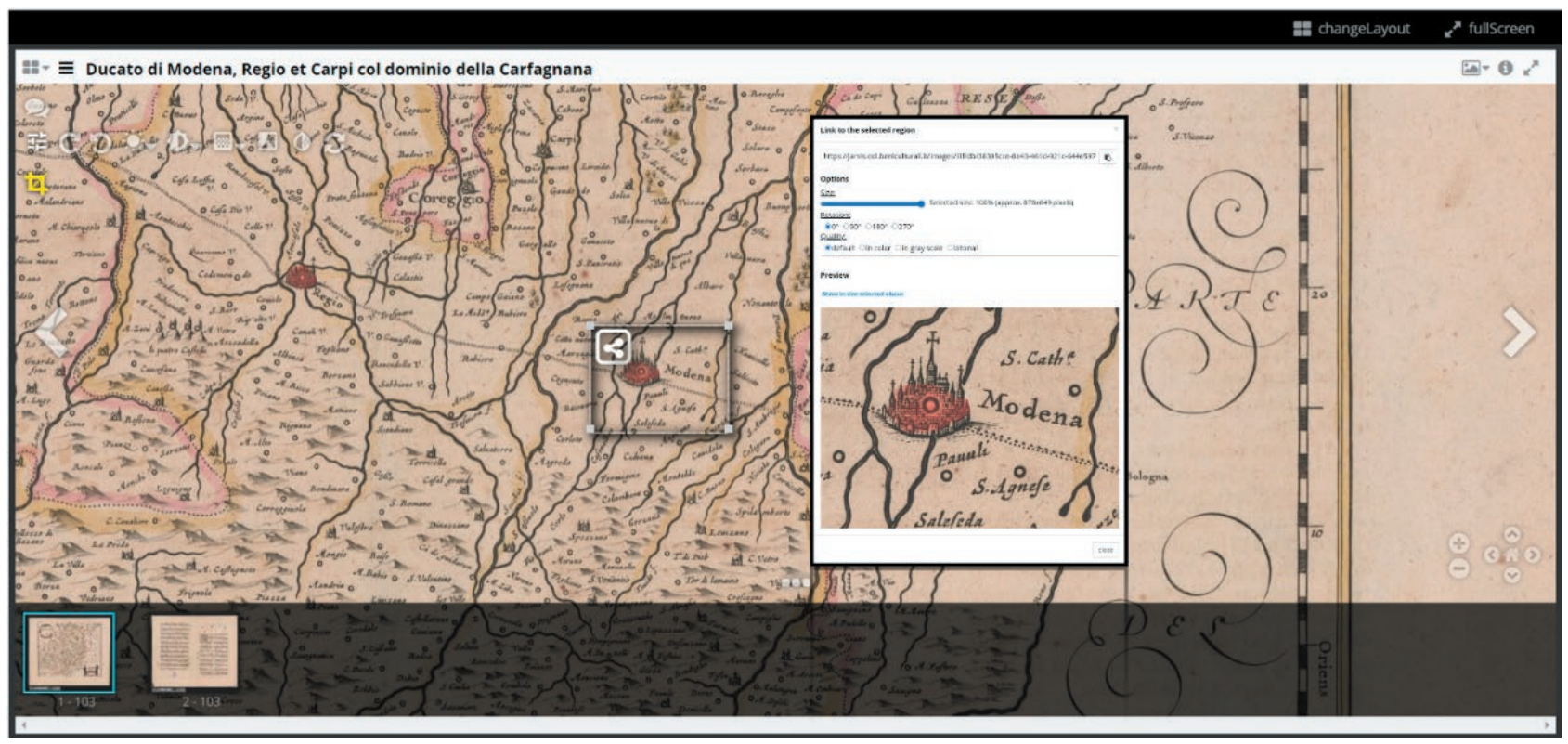

Figura 3. Esempio di utilizzo dello strumento "Ritaglia". Fonte: Estense Digital Library.

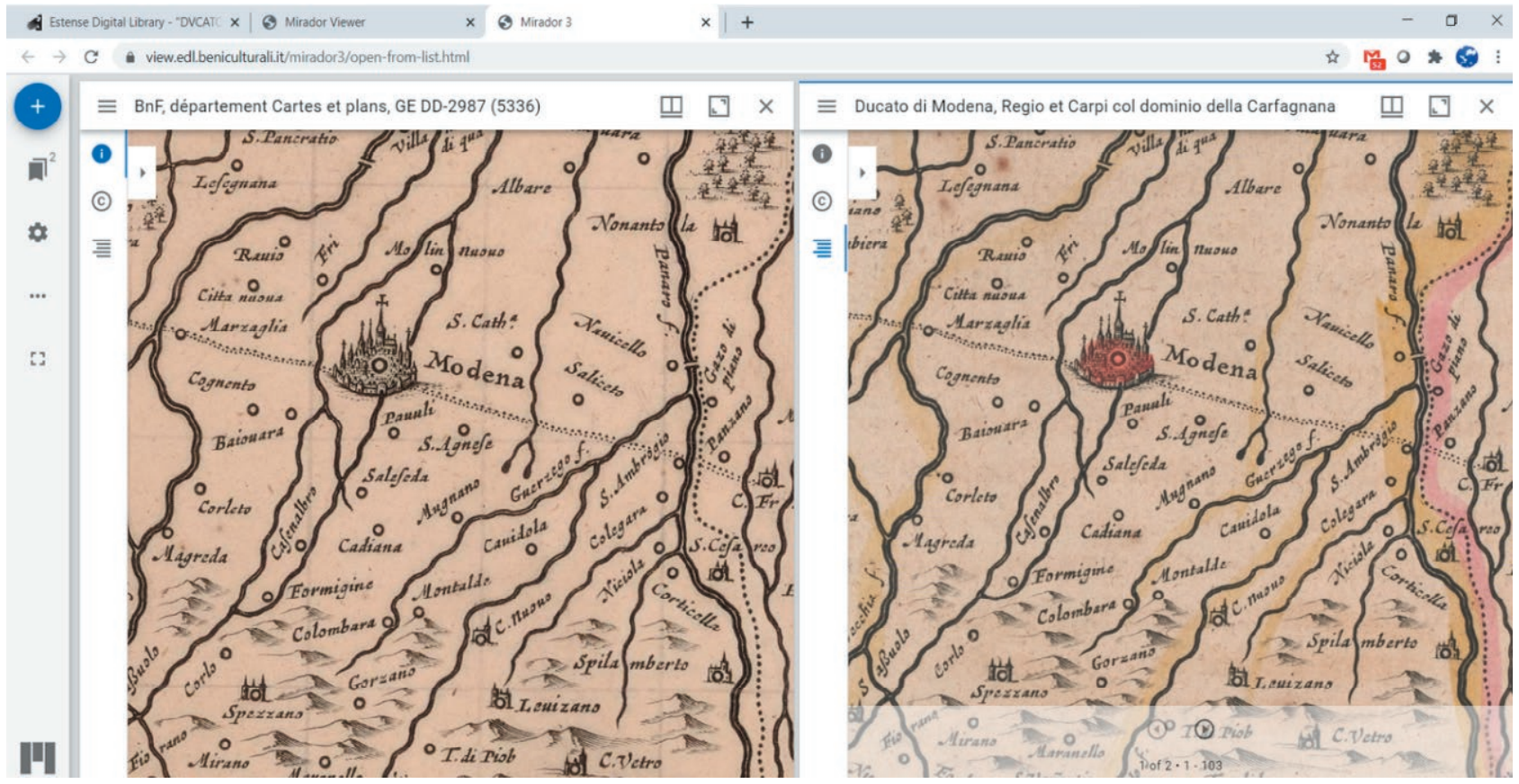

Figura 4. Esempio di comparazione tra due mappe: la prima posseduta dalla BEU, la seconda dalla Bibliothèque Nationale de France. Fonte: Estense Digital Library.

nel nostro caso di una mappa, a cui associare brevi testi descrittivi. L'opzione Crea nuova storia, nella pagina Le mie storie, accessibile dal menu Account, permette, così, di aprire un editor in cui inserire il titolo del progetto, una breve descrizione e il nome dell'autore. A destra del- lo schermo è poi possibile aggiungere i documenti desiderati, selezionabili dal Catalogo Estense o dal Catalogo Open, attraverso il cosiddetto manifest, ossia un link che consente di inserire nella propria storia l'immagine selezionata. A questo punto si può creare un vero $\mathrm{e}$ 
proprio storytelling selezionando i dettagli che si vogliono mostrare in uno o più documenti, a fianco dei quali vengono aggiunti un titolo e una breve descrizione. La presentazione finale è organizzata con una serie di slide che scorrono sullo schermo muovendo la visualizzazione all'interno dei documenti a differenti zoom e su diversi dettagli, facendo susseguire al contempo i testi relativi a ogni inquadratura, raccontando la propria storia. Anche in questo caso le storie possono essere create in modalità privata oppure condivise con gli utenti ${ }^{27}$.

\section{Conclusioni}

Come abbiamo avuto modo di vedere nel precedente paragrafo, l'Estense Digital Library ha contribuito al miglioramento del lavoro dei ricercatori sul patrimonio BEU innanzitutto perché ha creato un catalogo unico, che consentirà la valorizzazione di un patrimonio poco conosciuto alla stessa BEU, che ha come propri vessilli tre importantissime mappe (Cantino, Catalana e Castiglioni), ma ha "dimenticato" per lungo tempo altre mappe custodite nei cassetti e nei camerini di Palazzo dei Musei. Il nuovo catalogo, quindi, ha consentito di "riportare" alla luce la collezione di mappe mettendole a disposizione degli studiosi tramite un'interfaccia online, completa, che permette la ricerca tramite parole chiave e la consultazione senza doversi preoccupare della fragilità del documento e della sua dimensione. A ciò si aggiungono le funzioni di IIIF che, come abbiamo visto, facilitano la comparazione, l'annotazione e la condivisione del lavoro. Non solo, questi strumenti hanno un potenziale molto interessante anche in campo didattico, poiché forniscono da un lato la possibilità al docente di creare degli storytelling direttamente sul documento digitalizzato, dall'altro permette agli studenti stessi di utilizzare gli strumenti per svolgere approfondimenti sui temi affrontati. Questa potenzialità consente non solo di affrontare in modo innovativo $\mathrm{i}$ temi di studio, ma favorisce anche una valorizzazione e una consapevolezza del patrimonio della BEU nel pubblico e in particolare nelle generazioni che nel futuro saranno chiamate a conservarlo.

Il progetto portato avanti dalla BEU, però, non ha avuto solo il merito di creare strumenti utili per la ricerca, ma ha anche stimolato la ricerca nel campo delle digital humanities, grazie al fiorire di progetti collaterali nati grazie alla collaborazione con Università di

\footnotetext{
${ }^{27}$ Per un approfondimento delle funzioni di IIIF e dell'Estense Digital Library si rimanda al sito del progetto e in particolare alla Guida delle funzionalità di EDL: https://edl.beniculturali.it/page/623.
}

Modena e Reggio Emilia e AGO Fabbriche Culturali, a cui partecipa anche Jeffrey Schnapp figura di riferimento in questo campo di studi. Tra le possibilità offerte da questa collaborazione emerge in particolare il lavoro svolto dall'AImageLab che sta utilizzando proprio le immagini digitalizzate di EDL per l'elaborazione di strumenti utili per l'OCR dei manoscritti e, nel futuro, delle mappe, rendendo la BEU e le Gallerie Estensi dei veri e propri laboratori di sperimentazione in campo di intelligenza artificiale, data mining e pattern recognition. Un altro progetto è Este Soundscape, nato dalla collaborazione tra gli assegnisti che si sono occupati dello studio della collezione cartografica e musicale con la collaborazione degli ingegneri, che propone la creazione di una piattaforma cartografica digitale attraverso la quale conoscere ed esplorare i luoghi, gli eventi e le persone che hanno contribuito alla costruzione del paesaggio sonoro della città di Modena ${ }^{28}$ nel XVII secolo (Fiore, Belotti 2020).

Il progetto, quindi, ha favorito l'incontro tra ricercatori di settori differenti che hanno iniziato a riflettere insieme sulle potenzialità e le applicazioni delle digital humanities aprendo nuove vie alla sperimentazione. Simbolo di tale lavoro di sviluppo interdisciplinare è la nascita del centro interdipartimentale DHMoRe dell'Università degli Studi di Modena e Reggio Emilia, che dal 2020 coordina i vari progetti in corso, favorendo proprio l'incontro e il dialogo tra ingegneri e umanisti, per lo sviluppo di una nuova cultura umanistica digitale.

\section{Riferimenti bibliografici}

Almagià, R. (1926). Notizie di quattro carte nautiche della R. Biblioteca Estense. La Bibliofilia. Rivista di storia del libro e delle arti grafiche di bibliografia ed erudizione, 27, 337-347.

Bagnoli, M., Battini, A. (2018). Meravigliose avventure. Racconti di viaggiatori del passato. Modena, Panini.

Bertoni, G. (1903). La Biblioteca Estense e la coltura ferrarese ai tempi del duca Ercole I (1471-1505). Torino, Loescher.

Bonazzi, A., Dameri, D., Farinelli, F., Lodovisi, A., Torresani S. (a cura di) (1995). Giacomo Cantelli. Geografo del Serenissimo. Casalecchio di Reno (BO), Grafis Edizioni.

\footnotetext{
${ }^{28} \mathrm{Nel}$ corso del Seicento, Modena divenne un importante centro culturale e musicale grazie alla famiglia Este. In particolare, durante il regno di Francesco II d'Este (1674-94) la produzione musicale conobbe la sua fase più significativa. Alcune istituzioni cittadine furono partecipi dellevoluzione stessa del linguaggio musicale, influenzando le modalità di produzione e di fruizione della musica.
} 
Casti, E. (1998). Lordine del mondo e la sua rappresentazione. Semiosi cartografica e autoreferenza. Milano, Unicopli.

Cassella, M. (2017). Biblioteche di ricerca e digital humanities. Biblioteche Oggi, 17, 44-51.

Comune di Modena (2009). La 'Rappresentazione' della Città di Modena. Gian Battista Boccabadati. Poeta, letterato, cartografo. Quaderni dell'Archivio Storico di Modena, XXIV. Modena, Fondazione C.R.M.

Di Giammarco, F. (2019). Lo standard IIIF per lo sviluppo delle biblioteche digitali, 13 novembre 2019. https://www. culturadigitale.it/biblioteca-digitale/617/lo-standard-iiifper-lo-sviluppo-delle-biblioteche-digitali/

Donattini, M. (2000). Cultura geografica ferrarese del Rinascimento. In Prosperi, A. (a cura di). Storia di Ferrara, vol. VI: Il Rinascimento. Situazioni e personaggi. Ferrara, Corbo, 407-58.

Fiore, A., Belotti, S. (2020). Merging music and landscape: a digital approach to discover the cultural identity of early modern Modena. Magazén. International Journal for Digital and Public Humanities, 1 (1), 75-100. DOI: http:// doi.org/10.30687/mag//2020/01/004

Frabetti, P. (1978). Carte nautiche italiane dal XIV al XVII secolo conservate in Emilia-Romagna. Archivi e biblioteche pubbliche. Firenze, Olschki.

Lamberti, A., Tammaro, A.M. (2020). Come cambiano nel mondo i servizi delle biblioteche per il Coronavirus. Bibelot: notizie dalle biblioteche toscane, 26 (1). https:// riviste.aib.it/index.php/bibelot/article/view/12030

Mattei, M.G. (a cura di) (2015). Jeffrey Schnapp. Digital humanities. Milano, Egea.

Milano, E. (1991). La Carta del Cantino e la rappresentazione della Terra nei codici e nei libri a stampa della Biblioteca Estense e Universitaria. Modena, Il Bulino.

Milano, E. (1995). Il Mappamondo Catalano Estense. In Milano, E., Battini, A. (a cura di), Il Mappamondo Catalano Estense, Dietikon-Zürich, Urs Graf Verlag, 7-63.

Milano, E. (2001), Alla scoperta del mondo. L'arte della cartografia da Tolomeo a Mercatore. Modena, Il Bulino.

Milano, E., Battini, A. (2002). Planisfero Castiglioni: Carta del navegare universalissima et diligentissima: 1525 . Modena, Il Bulino.

Palazzi, A. (2010). Il trattamento delle immagini digitali alla Biblioteca estense universitaria di Modena (19902010). DigItalia. Rivista del digitale nei beni culturali, 2, 62-78.
Salmson, J., Moretti, W. (a cura di) (1984). The Renaissance in Ferrara and its European Horizons. Rinascimento a Ferrara e i suoi orizzonti europei. Ravenna, Edizioni del Girasole.

Sgambati, G. (2010). Cooperazione fra le istituzioni per la catalogazione, la digitalizzazione e la valorizzazione del materiale cartografico. In Carta, M., Spagnoli, L. (a cura di). La ricerca e le istituzioni tra interpretazione e valorizzazione della documentazione cartografica. Roma, Gangemi, pp. 195-209.

Tinti, P. (2014). Itinerari di una raccolta libraria e dei suoi incunaboli. I libri di Matteo Pagliaroli all'Estense di Modena. In Bertolini, L., Coppini, D., Marsico, C. (a cura di). Nel cantiere degli umanisti. Per Mariangela Regoliosi. Firenze, Edizioni Polistampa, pp. 1257-1290.

Zanni. A. (2019), Il progetto di digitalizzazione della Biblioteca Estense Universitaria di Modena. Biblioteche oggi, 37, 63-67. 